\title{
Epidermolytic Acanthoma Mimicking Condyloma: A Case Report
}

\author{
Amandine Dupont $^{a} \quad$ Hélène Marescassier $^{b}$ Gürkan Kaya ${ }^{a, b}$ \\ a Department of Clinical Pathology, University Hospital of Geneva, Geneva, Switzerland; \\ ${ }^{b}$ Department of Dermatology and Venereology, University Hospital of Geneva, Geneva, \\ Switzerland
}

\section{Keywords}

Epidermolytic acanthoma $\cdot$ Condyloma acuminate $\cdot$ Histology

\begin{abstract}
Epidermolytic acanthoma is a rare benign tumor that appears as a solitary papule or, rarely, multiple small papules on the trunk and extremities, or on genitalia. They are generally asymptomatic, although they can be pruritic. The clinical presentation is often misleading, and the lesions are often misdiagnosed histologically and frequently confused with condyloma acuminatum. Here, we report a case of an epidermolytic acanthoma on the penis of a 57-yearold male, whose final diagnosis was made after several years.

\section{Introduction}

Epidermolytic acanthoma (EA) is a rare distinctive benign tumor originating in the epidermis that can be the source of clinical and histological misdiagnosis. The lesion is clinically a skin-colored or whitish papule and the surface can be verruciform [1]. It occurs in a wide range of individuals including young children and elderly adults, with male predominance [2]. The lesion is found on genitalia (scrotum and vulva) or on the trunk and extremities and can be solitary or multiple. The cause of EA is generally unknown and multiple reports have failed to prove the presence of human papilloma virus (HPV) [3]. No treatment is usually necessary, unless it causes disturbing symptoms or cosmetic concerns in the individual. 
Histologic distinction between EA and other squamous proliferations poses a common diagnostic challenge to pathologists and clinicians. Here, we present a case of an EA misdiagnosed for several years as a condyloma acuminatum (CA).

\section{Case Presentation}

A 51-year-old male known for psoriasis and treated with adalimumab (Humira ${ }^{\circledR}$ ) since 2002 , presented a few asymptomatic papules of the glans penis since 2008. The first biopsy concluded a CA and the patient was treated with cryotherapy, with no significant benefit. During the last physical examination in September 2018, 4 whitish papules of the glans penis were still present (Fig. 1). The differential diagnosis was clinically CA versus intraepithelial neoplasm versus psoriasis. The patient had stopped his treatment of adalimumab 6 months previously and in spite of that, the psoriasis was clinically mild with a PASI score of 2.5. A new biopsy of the penis was performed. Histologically, dysplasia could be excluded. Acanthosis and hyperkeratosis with variable degree of papillomatosis were present (Fig. 2a). The keratinocytes of the granular and spinous layer showed cytoplasmic vacuolization, coarse keratohyaline granules, and eosinophilic inclusions, consistent with EA (Fig. 2b, c). The review of the first biopsy in 2008 diagnosed as CA showed the same histological image and allowed the same conclusion. As the patient did not experience any inconvenience, no further treatment was continued.

\section{Discussion/Conclusion}

Both CA and EA are verrucous tumors that are sometimes difficult to distinguish one from the other clinically and histologically.

EA is a distinctive lesion first described by Shapiro and Baraf [4] in 1970, that occurs predominantly on the trunk, extremities, or scrotum and vulva of elderly adults (fifth to seventh decade) [1]. It appears clinically as small white or fleshy papules varying between 1 and $2 \mathrm{~mm}$. They can be solitary or multiple [5,6]. The disseminated form most frequently affects genital skin. They are often asymptomatic but can be pruritic, and the chronic form can cause lichenification [2, 7].

The histopathologic features are acanthosis and hyperkeratosis with variable degree of papillomatosis [1]. Ackerman [8] described EA 50 years ago as a combination of variously sized clear spaces around nuclei in the stratum spinosum and granulosum, amphophilic material forming indistinct cellular boundaries, markedly thickened granular zone containing keratohyalin bodies, and compact hyperkeratosis. This pattern differs from the cytopathogenic effect of HPV, where a clear halo around the nucleus is homogenous and the nucleus is retracted. Acantholysis is also different, as cytoplasm is eosinophilic and the nucleus is hyperchromatic [2]. Four histological patterns of EA have been described, which may coexist in the same lesion. They consist of the papillomatous, cup-shaped, acanthomatous, and polypoid variants. Ultrastructural studies have shown tonofilament alterations with agglutination of them in the cytoplasm and intracellular vacuolization, as well as premature cornification of keratinocytes [2, 7].

EA is part of the epidermolytic hyperkeratosis family, which is characterized by compact hyperkeratosis with granular and vacuolar degeneration. This histological pattern can be seen in a number of various clinical settings, such as in the vicinity of various tumors or inflammatory lesions, as well as in hereditary diseases such as keratinopathic ichtyosis or Vörner's palmoplantar keratoderma, epidermolytic epidermal nevi, and acrosyringial epidermolytic papulosis neviformis $[2,3]$. The clinical presentation is therefore the key to identifying the correct diagnosis. 
Fig. 1. Multiple small white papular lesions on the penis.
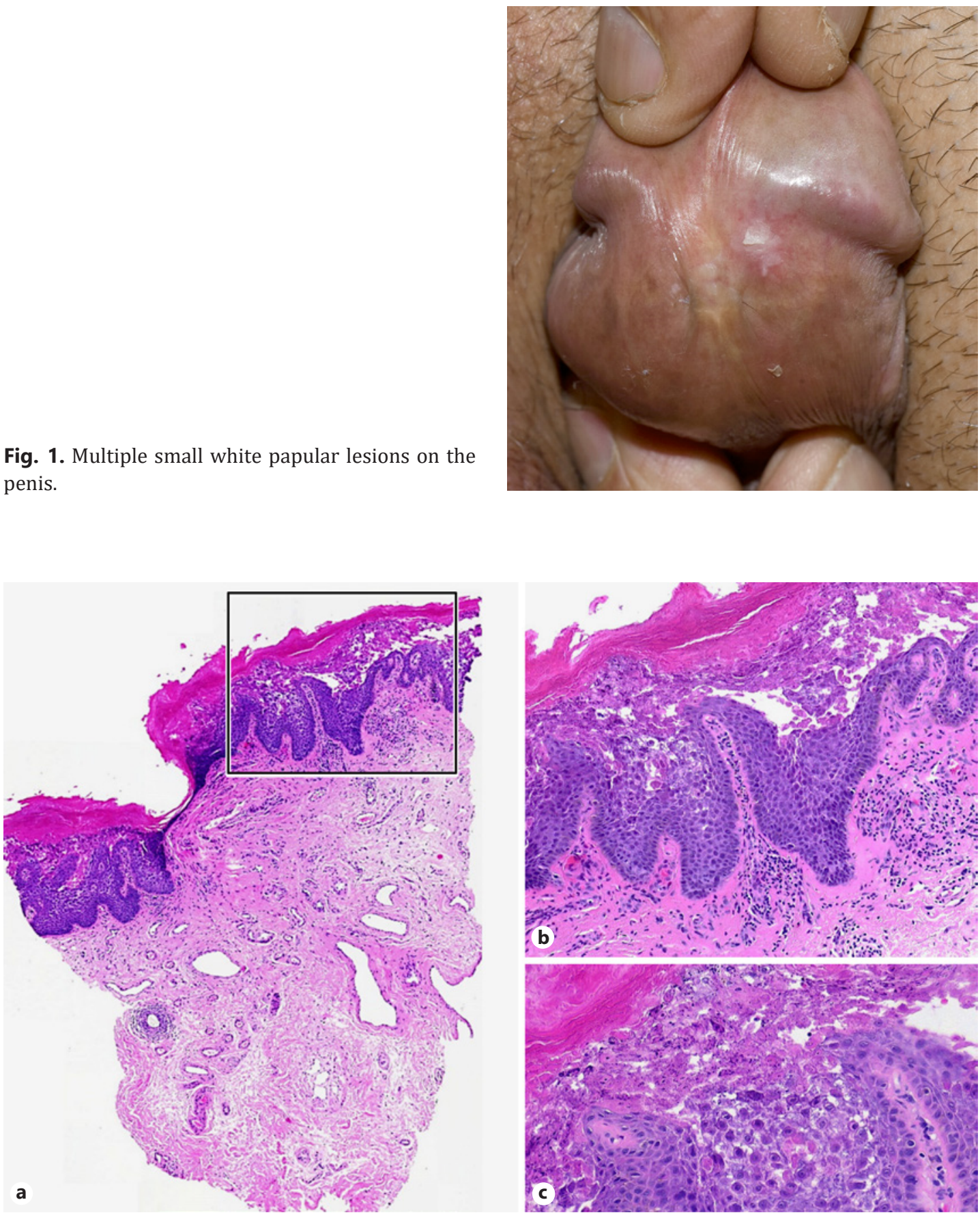

Fig. 2. a Whole-mounted view. b Marked hyperkeratosis with acanthosis and papillomatosis. c The keratinocytes of the granular and spinous layer show cytoplasmic vacuolization, coarse keratohyalin granules, and eosinophilic inclusions.

The precise etiology of the lesion is not known. Some have suggested that EA may be a local variant of the generalized hereditary epidermolytic hyperkeratosis. By analogy with this hereditary disorder of keratinization, mutations in KRT1 and KRT10 genes have been searched, but with no conclusive results $[9,10]$. Some other have looked for a link between 
EA and HPV, with no consistent result. The molecular studies have failed to detect a viral genome, and immunostainings for p16 were consistently negative in solitary as well as multiple lesions $[3,11]$. EA generally occurs in patients with no previous sexually transmitted disease and with no risky sexual behavior [2]. Trauma or chronic scratching has also been suggested without robust evidence. Some stated the hypothesis of immunosuppression [11]. No treatment is needed, but if the patient is embarrassed, various therapies can be used, including surgical excision, cryotherapy, or use of topical immune system modulators, with variable response rates [3].

In contrast, CA occurs predominantly on the genitalia of young adults and are HPV induced. They are clinically defined as fleshy exophytic lesions, often larger that EA. Lesions can be present on the glans penis, penile shaft, penile root, pubic area, groin, and perianal area, in contrast to EA which occurs more often on the scrotum in men or in the labia majora in women [3]. The histological pattern resembles that of EA as there are also acanthosis, papillomatosis, and hyperkeratosis. Coarse keratohyalin granules may also be seen. However, acantholysis is usually not present and, as mentioned previously, a clear halo around the nucleus is homogenous and the nucleus is retracted.

The clinical presentation of EA is misleading and misdiagnosis can easily occur, especially because this is a rare entity. In a series of 60 patients, Kazlouskaya et al. [12] showed that none of them were initially correctly diagnosed clinically. As an example, here we report the case of a patient whose diagnosis was first CA and needed several years to be corrected to EA.

In conclusion, recognition of this type of lesion is important as an incorrect CA diagnosis could cause unnecessary psychosocial anxiety [11] and as treatment is not necessary for EA due to the usually small size and its indolent nature. Overtreatment can be avoided and surgical excision may be proposed for cosmetic concerns.

\section{Statement of Ethics}

The patient gave his informed consent. The authors have no ethical conflicts to disclose.

\section{Disclosure Statement}

The authors have no conflicts of interest to declare.

\section{References}

1 Chan MP. Verruciform and Condyloma-like Squamous Proliferations in the Anogenital Region. Arch Pathol Lab Med. 2018 Sep [Epub ahead of print].

2 Moulonguet I. Multiple epidermolytic acanthomas of the genitalia. Ann Dermatol Venereol. 2017 Apr;144(4): 295-300. French.

3 Lee TJ, Wu YH. Multiple epidermolytic acanthomas mimicking condyloma: a retrospective study of 8 cases. Int J Dermatol. 2018 Jan;57(1):28-33.

4 Shapiro L, Baraf CS. Isolated epidermolytic acanthoma. A solitary tumor showing granular degeneration. Arch Dermatol. 1970 Feb;101(2):220-3.

5 Fletcher JW, Ramamurthi A, Parekh P. Presentation of epidermolytic acanthomas as multiple tan papules on the vulva. Proc Bayl Univ Med Cent. 2016 Apr;29(2):198-9.

6 Hijazi MM, Succaria F, Ghosn S. Multiple localized epidermolytic acanthomas of the vulva associated with vulvar pruritus: a case report. Am J Dermatopathol. 2015 Apr;37(4):e49-52.

7 Kukreja T, Krunic A. Multiple epidermolytic acanthomas must not be confused with genital human papillomavirus infection. Acta Derm Venereol. 2009;89(2):169-71. 
8 Ackerman AB. Histopathologic concept of epidermolytic hyperkeratosis. Arch Dermatol. 1970 Sep;102(3): 253-9.

9 Egozi-Reinman E, Avitan-Hersh E, Barzilai A, Indelman M, Bergman R. Epidermolytic Acanthoma of the Genitalia Does Not Show Mutations in KRT1 or KRT10. Am J Dermatopathol. 2016 Feb;38(2):164-5.

10 Cohen PR, Ulmer R, Theriault A, Leigh IM, Duvic M. Epidermolytic acanthomas: clinical characteristics and immunohistochemical features. Am J Dermatopathol. 1997 Jun;19(3):232-41.

11 Irwin S, Karim A, McHenry P, Hutchinson S, Miller K, Jamison J, et al. Multiple Epidermolytic Acanthomas: Rare Vulval Lesions Which May be Mistaken for Viral Warts. Int J Gynecol Pathol. 2018 Nov [Epub ahead of print].

12 Kazlouskaya V, Lambe J, Elston D. Solitary epidermolytic acanthoma. J Cutan Pathol. 2013 Aug;40(8):701-7. 